\title{
PAEDIATRIC ANTIPHOSPHOLIPID SYNDROME
}

\author{
Manole Cojocaru ${ }^{1}$, Inimioara Mihaela Cojocaru ${ }^{2}$ \\ 1"Titu Maiorescu University”, Faculty of Medicine, Department of Physiology, “Dr. Ion Stoia” Center for Rheumatic Diseases Bucharest, Romania \\ 2"Carol Davila" University of Medicine and Pharmacy, Department of Neurology, Colentina Clinical Hospital, Bucharest, Romania
}

\begin{abstract}
Antiphospholipid syndrome (APS) is a thrombotic disorder characterised by the association of arterial and venous thrombosis with antibodies directed against phospholipids. The onset of APS must occur before the patient's 18th birthday. Clinical manifestations were temporally correlated to the presence of antiphospholipid (aPL) antibodies if they occurred within 6 months. In contrast with adults, however, transient non-thrombocytogenic aPL antibodies are seen more commonly, usually after childhood infections. The presence of aPL antibodies, specifically aCL antibodies, was significantly associated with thrombotic events. Patients
\end{abstract}

with childhood-onset APS presented with significantly more episodes of chorea and jugular vein thrombosis than adults. The aPL antibodies are tested by coagulation or immunologic assays. The presence of a prolonged prothrombin time in the setting of lupus anticoagulant (LA) may be a marker of paediatric systemic lupus erythematosus (SLE). This review highlights recent clinical advances in the field of APS in children.

Key words: antiphospholipid syndrome, antiphospholipid antibodies, paediatrics
In recent years, antiphospholipid (aPL) antibodies and their associated clinical features have been increasingly recognised in various autoimmune and non-autoimmune diseases. Antiphospholipid syndrome (APS) can occur in children, as in adults, with the same diverse spectrum of thrombotic sites. APS is the most common acquired state of hypercoagulation in both adults and children. A close association between aPL antibodies and recurrent arterial and/or venous thrombosis has been supported by several retrospective and prospective studies, and it appears that aPL antibodies have a direct role in the pathogenesis of the thrombophilic state of APS (1-3). Recurrent thrombotic events seem less frequent than in adults $(4,5)$. The most common presentation in the paediatric population is stroke. Stroke in children is significantly less common than stroke in adults. Childhood stroke is uncommon but is an important cause of mortality and morbidity in children. The most common cause of ischaemic stroke in children is thrombotic vessel occlusion. Whereas APS in adults has been well characterised, only a few studies of children with APS have been reported (6). Paediatric patients with APS constitute a sufficient sample to investigate the relationship between aPL antibodies and the associated clinical manifestations, such as thrombocytopenia, haemolytic anaemia, chorea, livedo reticularis, and the specificities of aPL antibodies that are linked to thrombosis. The recently revised criteria for the diagnosis of APS in adults are currently used for paediatric patients, but there is no confirmation of these criteria for children (7).

Several tests for aPL antibodies are available. However, the prolonged activated partial thromboplastin time test has a low sensitivity; kaolin clotting time is too complicated to assay; andbut dRVVT (Russell viper venom time) is very sensitive and is currently the preferred test for lupus anticoagulant (LA) detection. Other tests for measuring aPL antibodies are immunological, namely the enzyme-linked immunosorbent assay, which detects different isotypes of antibodies to many other phospholipids (phosphatidylserine, phosphatidylinositol, phosphatidylcholine). Their specificity increases with the titer and the aCL IgG antibody is more specific than aCL IgM. Another group of antibodies is directed against protein co-factors 
that bind the phospholipids, such as beta-2 glycoprotein-I $(\beta 2 \mathrm{GPI})$ and annexin. It is important to use more than one test to detect them. The detection of persistently elevated levels of aPL antibodies is a requisite laboratory feature for the diagnosis $(8,9)$.

The laboratory criteria include : a positive LA test, the presence of medium or high titre aCL antibodies, or anti$\beta 2$ GPI IgG and IgM antibodies, on two occasions at least 12 weeks apart, or the presence of at least one type of autoantibody, known as an aPL antibody, in the plasma. Low titer $\mathrm{aCL}$ antibodies and anti- $\beta 2 \mathrm{GPI}$ antibodies have been reported in $11 \%$ and $7 \%$ of healthy children, respectively (10).

Serum levels of aCL IgA antibodies are lower in children than in adults, whereas the highest levels of anti- $\beta 2$ GPI IgG antibodies were found in preschool children $(11,12)$.

Persistent aPL antibodies are associated with an increased risk of arterial thrombosis (mainly cerebrovascular events) (13).

The prevalence and levels of aPL antibodies in children with SLE are higher than in healthy controls, with aCL antibodies ranging from $27-57 \%$ and LA ranging from 16-29\% $(14,15)$. The presence of an aPLantibody was significantly associated with anti-double-stranded DNA antibodies but was not significantly associated with neuropsychiatric manifestations or thrombocytopaenia. The presence of an aCL antibody was significantly associated with haemolytic anaemia. A prolonged prothrombin time, in the setting of LA (all with a prolonged activated partial thromboplastin time), was associated with life-threatening disease. The presence of an aPL antibody, specifically an aCL antibody, was significantly associated with thrombotic events. The presence of a prolonged prothrombin time in the setting of LA may be a marker of a more serious disease in paediatric SLE. In paediatric SLE, $\mathrm{aCL}$ antibodies are frequently found, high levels of aCL IgG antibodies are often associated with central nervous system involvement, and aCL antibodies have a low predictive value in the development of vascular thrombosis (16).

With the exception of stroke, anti- $\beta 2$ GPI antibody detection does not improve identification of paediatric APS over that of traditional aPL assays. Anti- $\beta 2$ GPI antibodies are rare in paediatric primary APS, but may predict the evolution of chronic thrombocytopenia to SLE (17).

Deep vein thrombosis of the lower limbs is the most common thrombosis in paediatric APS patients. Arterial thrombosis is much more common in children than in adults (7).

A wide variety of central nervous system symptoms in APS has been described, including chorea, dementia, migraine, intracranial hypertension, neurocognitive deficits, psychosis, depression, epilepsy, Guillain-Barré syndrome, transverse myelopathy, and optical neuritis $(18,19)$.

Cardiac disease is rare in children with APS. In a cohort of paediatric APS patients, thrombocytopenia and haemolytic anaemia were found in $10 \%$ of the patients. Livedo reticularis, the reticular cyanosis caused by vascular stasis of deep dermal vessels, is the most common skin manifestation of the syndrome, although it rarely appears in children. Renal and pulmonary involvement isare also rare in paediatric patients. In a cohort of 28 paediatric APS patients, the most common initial APS manifestations were venous thrombosis, stroke and thrombocytopaenia. Lupus anticoagulant was detected in almost all of the patients. Recurrent thrombotic events were less common in patients who received anticoagulant therapy. A high rate ( $45 \%$ of patients) of inherited thrombophilias was found in our series, implying that aPL antibodies may serve as a "second vascular hit" in children. Positive aPL antibody tests were observed in most infants and the clinical presentation consisted of arterial and venous thrombi in multiple localisations, which is similar to adult APS patients. In a cohort of children with APS, five patients presented with perinatal stroke (6).

In a review of 115 patients with catastrophic APS, only 3 were children. Rare cases of perinatal thrombosis in infants born to mothers with APS or aPL antibodies have been reported (20).

Antiphospholipid syndrome is an important cause of acquired thrombophilia. The presence of a prolonged prothrombin time in the setting of LA may be a marker of more serious disease in paediatric SLE. This syndrome is the most common cause of acquired thrombophilia and is and is associated with either venous or arterial thrombosis, or both. The prevalence of thrombophilia markers is increased in children with stroke compared with control subjects. Furthermore, factor V Leiden and aPL antibodies contribute significantly to stroke occurrence (13).

In APS, skin involvement is often the first sign (livedo reticularis, ulcerations, digital gangrene, subungueal splinter haemorrhages, superficial venous thrombosis, thrombocytopaenic purpura, pseudovasculitic manifestations, extensive cutaneous necrosis, and primary anetoderma) (21).

Neonatal APS is a rare clinical entity. It is characterised by neonatal thrombotic disease that is due to the transplacental passage of maternal aPL antibodies (22).

Data from paediatric APS studies have confirmed that APL antibody-related thrombi in children are frequently associated with multiple APL antibody positivity and the concomitant presence of inherited prothrombotic disorders. Comparisons between paediatric patients with primary APS and APS that is associated with an underlying autoimmune disease revealed several differences. Children with primary APS were significantly younger and had a higher frequency of arterial thrombotic events, particularly cerebrovascular ischaemic events. In contrast, children with APS that was associated with an underlying autoimmune disease were significantly older and had a significantly higher frequency of venous thrombotic events that were associated with haematologic and skin manifestations (23).

However, because of the high frequency of infectious processes in early life, children may have a greater prevalence of non-pathogenic and transient aPL antibodies. Due to the rarity of aPL antibody-related thrombosis in children, its natural history and optimal management can be determined only through large, multicentre, controlled studies (2). 
We recommend that all children with idiopathic ischaemic stroke should be completely investigated and evaluated for aPL antibodies. Due to the rarity of APS in children, multicentre prospective studies are warranted.

\section{CONCLUSION}

Antiphospholipid syndrome is considered to be the most commonly acquired hypercoagulation state of autoimmune disorders in children. The clinical and laboratory characterisation of patients with paediatric APS imply several important differences between APS in paediatric and adult populations. Children with APS have frequently demonstrated associated nonthrombotic manifestations, particularly haematological, skin and neurological manifestations. APS in children occurs predominantly with deep vein thrombosis and stroke. SLE may develop in a significant percentage of girls presenting with APS . Antiphospholipid antibodies reflect the lower prothrombotic haemostatic state of childhood.

\section{CONFLICTS OF INTEREST}

The authors have no conflicts of interest to declare.

\section{REFERENCES}

1. Giannakopoulos B, Passam F, Rahgozar S, Krillis SA. Current concepts on the pathogenesis of the antiphospholipid syndrome. Blood 2007; 109: 422-30.

2. Ravelli A, Martini A. Antiphospholipid syndrome in pediatrics. Rheum Dis Clin North Am 2007; 33: 499-523.

3. Berkun Y, Kenet G. Pediatric antiphospholipid syndrome. IMAJ. 2008; 10: 45-7.

4. Avicin T. Antiphospholipid syndrome in children. Curr Opin Rheumatol 2008; 20: 595-600.

5. Soares Rolim AM, Castro M, Santiago MB. Neonatal antiphospholipid syndrome. Lupus 2006; 15: 301-3.

6. Hoppensteadt DA, Fabbrini N, Bick RL, Messmore HL, Adiguzel C, Fareed J. Laboratory evaluation of the antiphospholipid syndrome. Hematol Oncol Clin North Am 2008; 22: 19-32.

7. Muscal E, Brey R. Antiphospholipid syndrome and the brain in pediatric and adult patients. Lupus 2010; 19:406-11.

8. Hunt BJ. Pediatric antiphospholipid antibodies and antiphospholipid syndrome. Semin Thromb Hemost 2008; 34: 274-81.
9. Cimaz R, Descloux E. Pediatric antiphospholipid syndrome. Rheum Dis Clin North Am 2006; 32: 553-73

10. Cohen D, Berger SP, Steup-Beekman GM, Bloemenkamp KWM, Bajema IM. Diagnosis and management of the antiphospholipid syndrome BMJ 2010; 340: c2541.

11. Rodrigues CEM, Carvalho JF, Shoenfeld Y. Neurological manifestations of antiphospholipid syndrome. Eur J Clin Invest 2010; 40: 350-9.

12. Muscal E, Brey RL. Neurologic manifestations of the antiphospholipid syndrome: integrating molecular and clinical lessions. Curr Rheumatol Rep 2008; 10: 67-73.

13. Rodrigues CEM, Carvalho JF, Shoenfeld Y. Antiphospholipid syndrome as a neurological disease. Curr Rheumatol Rev 2010; 6: 12-7.

14. Suárez Alvarez LS, Hughes GR, Khamashta MA. Neurological manifestations of the antiphospholipid syndrome. Med Clin (Barc) 2005; 124: 630-3.

15. Muscal E, Brey RL. Neurological manifestations of the antiphospholipid syndrome: risk assessments and evidencebased medicine. Int J Clin Pract 2007; 61: 1561-8.

16. Miiesbah W. Neurologic symptoms as a feature of the antiphospholipid syndrome. Semin Thromb Haemost 2008; 34: 286-9.

17. Rodriguez V, Reed AM, Kuntz NL, Anderson PM, Smithson WA, Nichols WL. Antiphospholipid syndrome with catastrophic bleeding and recurrent ischemic strokes as initial presentation of systemic lupus erythematosus. J Pediatr Hematol Oncol 2005; 27: 403-7.

18. Schmugge M, Revel Vilk S, Hiraki L, Rand ML, Blanchette VS, Silvermugge ED. Thrombocytopenia and thromboembolism in pediatric systemic lupus erythematosus. J Pediatr 2003; 143: 666-9.

19. Descloux E, Durieu I, Cochat P, Vital Durand D, Ninet J, Fabien N, Cimaz R. Paediatric systemic lupus erythematosus: prognostic impact of antiphospholipid antibodies. Rheumatology 2008; 47: 183-7.

20. Avcin T, Silverman E. Antiphospholipid antibodies in pediatric systemic lupus erythematosus and the antiphospholipid syndrome. Lupus. 2007; 16: 627-33.

21. Sanna G, DC, Ruz D, Cuadrado MJ. Cerebral manifestations in the antiphospholipid (Hughes) syndrome. Rheum Dis Clin North Am 2006; 32: 465-90.

22. Boffa MC, Lachassinne E. Infant perinatal thrombosis and antiphospholipid antibodies: a review. Lupus 2007; 16: 634-41.

23. Abend NS, Licht DJ, Spencer CH. Lupus anticoagulant and thrombosis following Henoch-Schönlein purpura. Pediatr Neurol 2007; 36: 345-7. 